\title{
A predicting severity surgical scoring system and outcome in elderly patients undergoing anesthesia
}

Marta Gomes Campos ${ }^{1}$, Ana R Teles ${ }^{1}$, Luís Guilherme Casimiro', Bárbara Azevedo ${ }^{1}$, Daniela Cristelo ${ }^{1}$, Fernando Abelha ${ }^{1,2}$

\section{BACKGROUND}

A new scoring system that assesses perioperative patient statuses was developed to predict outcome and severity at the end of anesthesia.

The surgical apgar score and the American Society of Anesthesiologists physical status (ASA-PS) were combined into a single scale: SASA.

Goal of Study: We aimed to determine SASA score and its clinical outcomes in elderly patients undergoing anesthesia.

\section{MATERIALS AND METHODS}

Observational prospective study

- Inclusion criteria: Patients aged>60 years, submitted to elective surgery and admitted to Post Anaesthetic Care Unit (PACU)

- Exclusion criteria: Patients admitted to Intensive Care Unit (ICU) or unable to give informed consent.

Charlson comorbidity_-index(CCl)

Clinical Frailty Scale (CFS)

Evaluated before surgery(D0) and 30 days(D30) later;

Frailty was defined as a score $\geq 5$.

Lawton Instrumental Activities of Daily Living Scale (IADL) and World Health Organization Disability Assessment Schedule (WHODAS)

- Evaluated on D0, D30 and 3 months later(M3) to assess dependency and disability.

Intraoperative data Lowest HR

Lowest MAP

Estimated blood loss

SASA $=s A s+(6-A S A-P S) \times 2$.

\section{SASA $<13 \rightarrow$ patients with predicted poor outcome (PPO).}

Physiological and Operative Severity Score for the Enumeration of Mortality and Morbidity (POSSUM )

Statistic analysis: The Mann-Whitney test, Chi-square or Fisher's exact test were used for comparison.

\section{RESULTS}

$$
N=235 \text { patients }
$$

$32.3 \%$ PPO $(n=76)$

Table 1: Population characterization

\begin{tabular}{|cccc|}
\hline & PPO & Non PPO & $p=$ \\
\hline Age, mean \pm SD & $\mathbf{7 2 . 5 9 \pm 7 . 9 9}$ & $70.23 \pm 7.35$ & 0.037 \\
\hline $\begin{array}{c}\text { Gender, \%(n=) } \\
\text { Female } \\
\text { Male }\end{array}$ & $\begin{array}{c}59 \%(45) \\
41 \%(31)\end{array}$ & $\begin{array}{c}52 \%(82) \\
48 \%(77)\end{array}$ & 0.272 \\
\hline $\begin{array}{c}\text { ASA, \%(n=) } \\
\text { I / II } \\
\text { III / IV }\end{array}$ & $\begin{array}{lll} \\
\mathbf{7 4} \%(56)\end{array}$ & $26,4 \%(42)$ & \\
\hline & & & \\
\hline
\end{tabular}

Graph1: Comorbidities

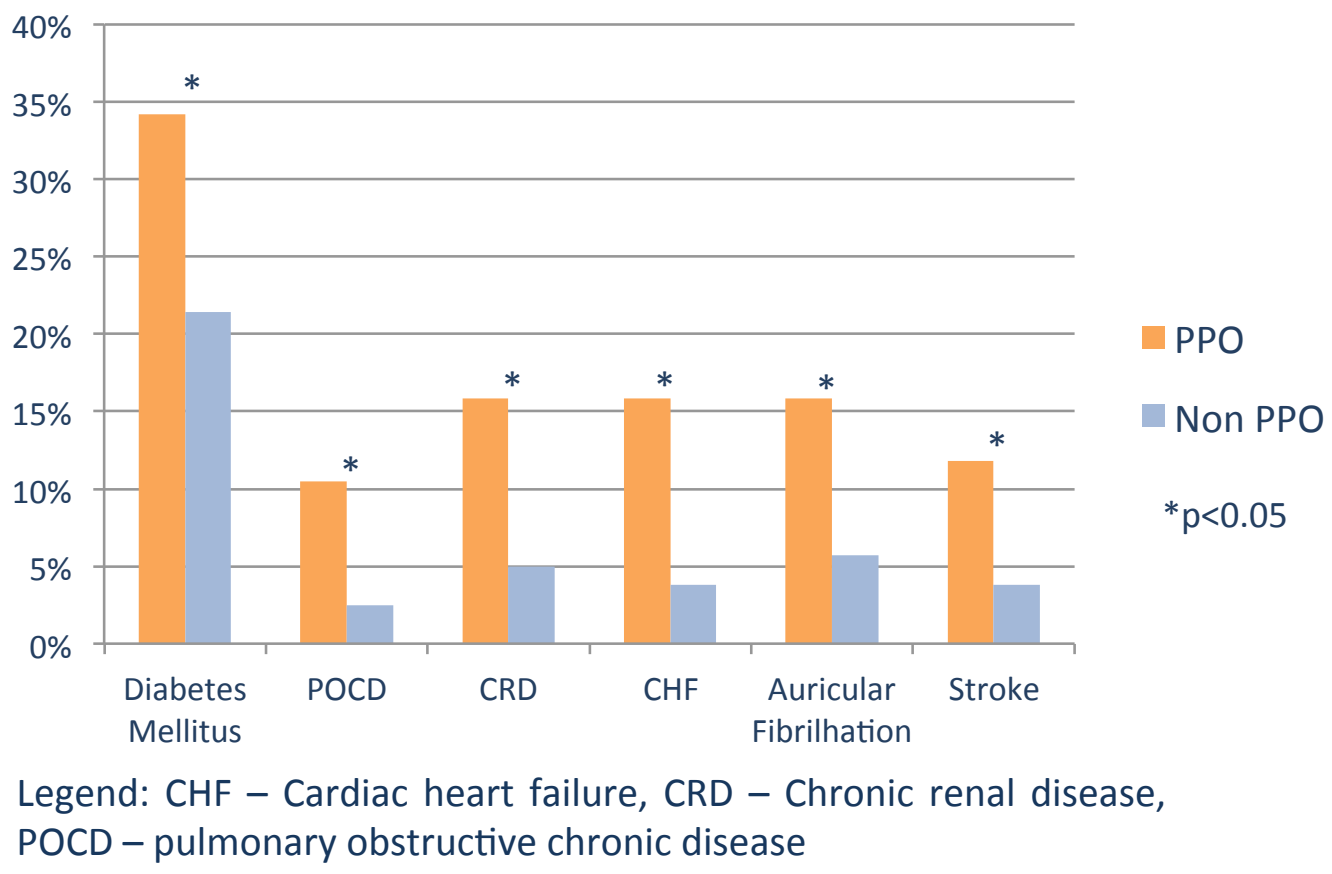
POCD - pulmonary obstructive chronic disease

Table 2: Pre and postoperative health state comparison by $\mathrm{CCI}, \mathrm{CFS}$, WHODAS and IADL.

\begin{tabular}{|cccc|}
\hline & PPO & Non PPO & $p=$ \\
\hline CCI (median) & 8 & 5 & 0.001 \\
\hline $\begin{array}{c}\text { Frailty (CFS) (\%) } \\
\text { D0 }\end{array}$ & $40 \%$ & $15 \%$ & $<0.001$ \\
M3 & $47 \%$ & $22 \%$ & $<0.001$ \\
\hline WHODAS (median) & & & \\
D0 & 29 & 8 & $<0.001$ \\
D30 & 31 & 8 & $<0.001$ \\
\hline IADL (median) & & & \\
D0 & 6 & 8 & $<0.001$ \\
M3 & 5 & 9 & $<0.001$ \\
\hline
\end{tabular}

Table 3: Intraoperative needs for coloids and blood infusion.

\begin{tabular}{|lccc|}
\hline & PPO & Non PPO & $p=$ \\
\hline Coloides infusion, \% (n=) & $9 \%(7)$ & $3 \%(4)$ & 0.041 \\
\hline Blood transfusion, \% (n=) & $8 \%(6)$ & $1 \%(2)$ & 0.014 \\
\hline
\end{tabular}

Table 4: Risk of morbidity and mortality by POSSUM.

\begin{tabular}{|cccc|}
\hline POSSUM & PPO & Non PPO & P \\
\hline Risk of morbidity & $40 \%$ & $34 \%$ & $<0.001$ \\
\hline Risk of mortality & $13 \%$ & $9 \%$ & $<0.001$ \\
\hline
\end{tabular}

Table 5: Duration of hospital stay.

\begin{tabular}{|cccc|}
\hline & PPO & Non PPO & $p=$ \\
\hline Hospital stay (days) & 8 & 4 & $<0.001$ \\
\hline
\end{tabular}

Graph 2: Reoperation and mortality rates.

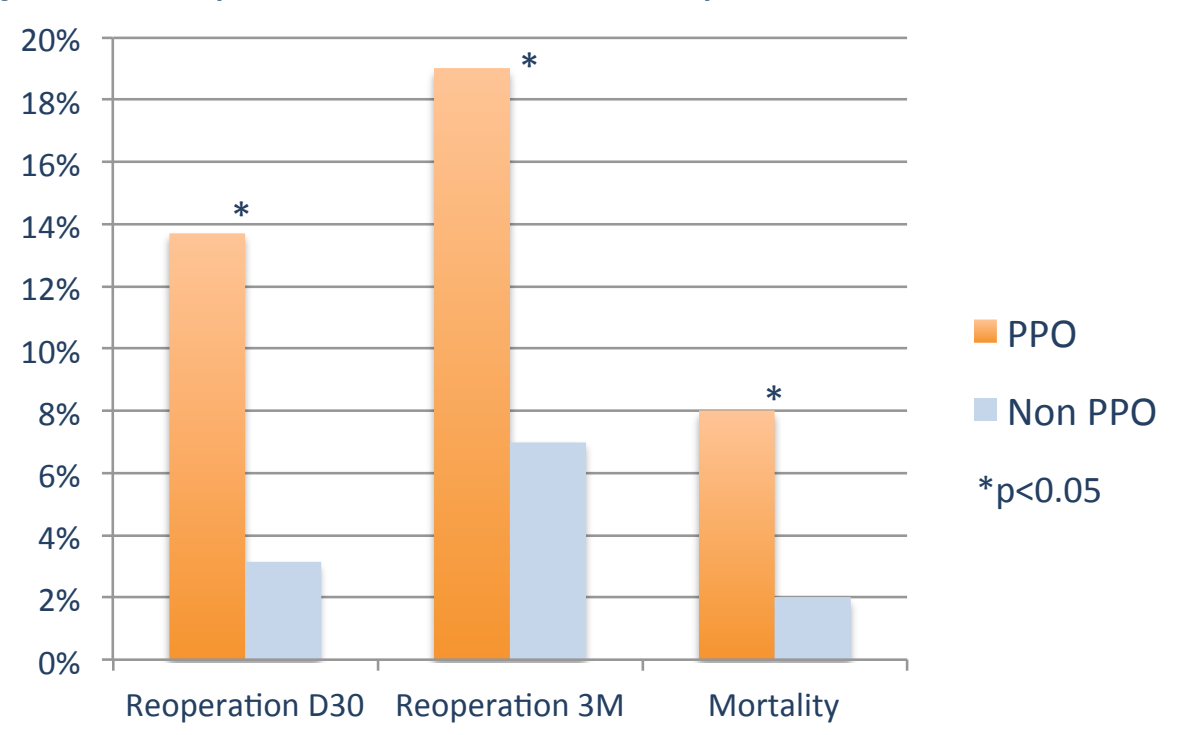

\section{CONCLUSIONS}

- In our study, patients with SASA score $<13$ were more dependent and frailer, with more comorbidities and blood transfusion needs.

- It was also related with higher hospital stay, mortality and reoperation rate in elderly patients. 